\title{
Multifunctional layer-by-layer carbon nanotube-polyelectrolyte thin films for strain and corrosion sensing
}

\author{
Kenneth J Loh ${ }^{1}$, Junhee Kim ${ }^{1}$, Jerome P Lynch ${ }^{1,2,5}$, \\ Nadine Wong Shi Kam ${ }^{3,4}$ and Nicholas A Kotov ${ }^{4}$ \\ ${ }^{1}$ Department of Civil and Environmental Engineering, University of Michigan, Laboratory for \\ Intelligent Structural Technologies (LIST), Ann Arbor, MI 48109-2125, USA \\ 2 Department of Electrical Engineering and Computer Science, University of Michigan, \\ Ann Arbor, MI 48109, USA \\ ${ }^{3}$ Michigan Society of Fellows, Horace H Rackham School of Graduate Studies, University of \\ Michigan, MI, USA \\ ${ }^{4}$ Department of Chemical Engineering, University of Michigan, Ann Arbor, MI 48109, USA \\ E-mail: jerlynch@umich.edu
}

Received 19 May 2006, in final form 14 December 2006

Published 9 February 2007

Online at stacks.iop.org/SMS/16/429

\begin{abstract}
Since the discovery of carbon nanotubes, researchers have been fascinated by their mechanical and electrical properties, as well as their versatility for a wide array of applications. In this study, a carbon nanotube-polyelectrolyte composite multilayer thin film fabricated by a layer-by-layer (LbL) method is proposed to develop a multifunctional material for measuring strain and corrosion processes. LbL fabrication of carbon nanotube composites yields mechanically strong thin films in which multiple sensing transduction mechanisms can be encoded. For example, judicious selection of carbon nanotube concentrations and polyelectrolyte matrices can yield thin films that exhibit changes in their electrical properties to strain and $\mathrm{pH}$. In this study, experimental results suggest a consistent trend between carbon nanotube concentrations and strain sensor sensitivity. Furthermore, by simply altering the type of polyelectrolyte used, $\mathrm{pH}$ sensors of high sensitivity can be developed to potentially monitor environmental factors suggesting corrosion of metallic structural materials (e.g. steel, aluminum).
\end{abstract}

(Some figures in this article are in colour only in the electronic version)

\section{Introduction}

During the past two decades, researchers have been optimizing and miniaturizing electronic devices for higher performance. For example, MEMS (microelectromechanical systems) technology allows complex circuitry, sensing and actuation mechanisms, and advanced computations to take place on a single microchip. As MEMS technology matures and performance limits are encountered, the field of nanotechnology brings forth promising new molecular tools and fabrication techniques that can improve the performance of current sensing devices. In particular, the discovery

5 Author to whom any correspondence should be addressed. of carbon nanotubes (CNTs) by Iijima in 1991 [1] led researchers to illustrate the use of carbon nanotubes in a wide variety of applications including nanocomposite materials [2], nanoelectronics [3], and various types of sensors [4, 5], among many others [6]. Their widespread adoption for novel applications arises from carbon nanotubes' impressive mechanical [7] and electrical properties [6].

Among the variety of sensing transducers used for structural health monitoring (SHM), strain and corrosion sensors rank among the most important for determining longterm structural reliability and health. For example, strain sensors are widely employed to measure component level strain in both laboratory and field environments, as they correspond directly to the induced localized stress fields 
Table 1. Matrix of 20 unique films fabricated.

\begin{tabular}{|c|c|c|c|c|}
\hline & & $0.25 \mathrm{mg} \mathrm{ml}^{-1} \mathrm{SWNT}$ & $0.50 \mathrm{mg} \mathrm{m}^{-1} \mathrm{SWNT}$ & $0.80 \mathrm{mg} \mathrm{ml}^{-1} \mathrm{SWNT}$ \\
\hline \multirow[t]{3}{*}{ Strain sensing } & u-SWNT-PSS/PVA ${ }^{\mathrm{a}}$ & 50 and 100 bilayers & 50 and 100 bilayers & 50 and 100 bilayers \\
\hline & p-SWNT-PSS/PVA ${ }^{b}$ & 50 and 100 bilayers & 50 and 100 bilayers & 50 and 100 bilayers \\
\hline & p-DWNT-PSS/PVA ${ }^{c}$ & 50 and 100 bilayers & 50 and 100 bilayers & 50 and 100 bilayers \\
\hline pH sensing & p-SWNT-PSS/PANI ${ }^{\mathrm{d}}$ & - & - & 50 and 100 bilayers \\
\hline
\end{tabular}

${ }^{a}$ Unpurified SWNTs dispersed in $1.0 \mathrm{wt} \%$ wt PSS with $1.0 \mathrm{wt} \%$ PVA LbL counterpart.

${ }^{\mathrm{b}}$ Purified SWNTs dispersed in $1.0 \mathrm{wt} \%$ PSS with $1.0 \mathrm{wt} \%$ PVA LbL counterpart.

${ }^{c}$ Purified DWNTs dispersed in $1.0 \mathrm{wt} \%$ PSS with $1.0 \mathrm{wt} \%$ PVA LbL counterpart.

${ }^{\mathrm{d}}$ Purified SWNTs dispersed in $1.0 \mathrm{wt} \%$ PSS with $1.0 \mathrm{wt} \%$ PANI LbL counterpart.

commonly used for damage detection. Consequently, since the development of the first strain gauges in 1936 [8], many researchers have followed suit to improve and design a broad array of strain transducers such as semiconducting strain sensors and fiber optic Bragg gratings. While semiconductor and fiber optic strain sensors can achieve high sensitivities and are generally easy to use, they can suffer from high manufacturing costs and require costly data acquisition systems. For corrosion sensing, many have proposed the use of $\mathrm{pH}$ sensors to monitor environmental factors favorable to the onset of corrosion. For example, passivation of reinforcing steel in concrete structures is strongly correlated to $\mathrm{pH}$; longterm $\mathrm{pH}$ monitoring can be used to detect the onset of corrosion, thus allowing for condition-based maintenance (as opposed to current schedule-based maintenance, which is more conservative and economically inefficient). Yet among the multitude of $\mathrm{pH}$ sensors available, current devices typically do not meet the geometric constraints and long-term durability needs of in situ corrosion monitoring. By adopting the tools and processes under development in the nanotechnology domain, one can tailor composite thin film materials with macro-scale electromechanical and electrochemical properties ideally suited for strain and corrosion sensing.

Early experimental investigations of CNTs as mechanical sensors have been presented by Peng et al [9]. These researchers quantify changes in the electrical properties of single-walled carbon nanotubes (SWNTs) as atomic force microscope (AFM) tips deflect SWNTs suspended over patterned silicon oxide/silicon substrate trenches. Their results indicate dramatically decreasing SWNT conductance of individual carbon nanotubes as AFM tips bend them [9-11]. Dharap et al [12] also reports on the change in resistance of CNT films, called 'buckypaper', as they are strained in low-tension-compression cycles. Similarly, Kang et al [13] has developed an SWNT-polymer composite buckypaper exhibiting linear piezoresistivity up to strains of $500 \mu \mathrm{m} \mathrm{m}^{-1}$.

In order to enhance the sensitivity and accuracy of traditional absorption-based $\mathrm{pH}$ sensors [14], Sawada et al [15] and continued efforts by Hizawa et al [16] have proposed a novel $\mathrm{Si}_{3} \mathrm{~N}_{4}$ hydrogen-ion-sensitive thin film coupled with charge coupled device (CCD) technology for two-dimensional $\mathrm{pH}$ sensing. Their $2 \mathrm{D} \mathrm{pH}$ image sensor successfully demonstrates real-time $\mathrm{pH}$ sensing of high sensitivity $(50.9 \mathrm{mV} / \mathrm{pH})$ and linear performance from $\mathrm{pH} 1$ to 14 . To bridge the gap between MEMS and nanotechnology, Kwon et al [17] utilizes SWNTs in a spray fabrication method over patterned silicon trenches for hydroxyl ion $\left(\mathrm{OH}^{-}\right)$ sensing. They have shown that as $\mathrm{OH}^{-}$groups are adsorbed onto SWNT walls the density of states at the Fermi level $\left(E_{\mathrm{F}}\right)$ increases, while the bandgap $\left(E_{\text {gap }}\right)$ drastically decreases. Although changes in sensor conductance are nonlinear and small at low $\mathrm{pH}$ values (1-5), conductivity change is enhanced at higher $\mathrm{pH}$ values (7-11), making them highly suitable for corrosion monitoring in concrete structures [18]. Recently, Kaempgen and Roth [19] have reaped the benefits of combining conductive polymers (i.e. polyaniline) with SWNTs to fabricate a transparent and conformable $\mathrm{pH}$ sensing transducer. They spray a solution of SWNT dispersed in $1.0 \%$ SDS (sodium dodecyl sulfate) onto polyterephthalate to produce a $\mathrm{pH}$ sensor exhibiting fast response times while showing excellent linearity across $\mathrm{pH}$ values ranging from 1 to 13 .

While many have proposed CNT based strain and $\mathrm{pH}$ transducers, few have developed a scheme capable of integrating different sensing transduction mechanisms within the same material. Furthermore, most of the existing work involves manipulating individual nanotubes for nano/micro-scale strain and $\mathrm{pH}$ sensing $[9-11,16,17,20]$. In this study, a layer-by-layer (LbL) assembly methodology is employed to manufacture a multifunctional homogeneous CNT-polyelectrolyte (PE) composite thin film from which strain and $\mathrm{pH}$ sensors can be produced. Unlike the buckypaper (made by drying CNT) employed by Dharap et al and Kang et al [12, 13], the CNT-PE thin films used here are multiphase composite systems consisting of nanotubes interwoven within a polyelectrolyte matrix. Unlike buckypaper, Mamedov et al [21] has shown that LbL films fabricated with polyethylenimine and polyacrylic acid have an ultimate tensile strength of approximately $200 \pm 40 \mathrm{MPa}$ and an ultimate strain capacity up to $10000 \mu \mathrm{m} \mathrm{m}^{-1}$. LbL thin films' inherently high mechanical strength makes them ideally suited for field instrumentation in structural components undergoing extreme loading. In an attempt to control the macro-scale sensitivity of CNT-PE thin-film electrical changes to strain or $\mathrm{pH}$ stimuli, fabrication parameters (i.e. CNT and PE concentrations) and the type of CNT (i.e. unpurified SWNTs, purified SWNTs, and purified double-walled carbon nanotubes (DWNTs)) are experimentally varied to quantify changes in the performance characteristics of CNT-PE strain and pH sensors (table 1).

\section{Experimental details}

\subsection{Layer-by-layer assembly}

Homogeneous multiphase carbon nanotube-polyelectrolyte composite thin films are fabricated via a systematic layerby-layer assembly technique [22-24]. The LbL method 


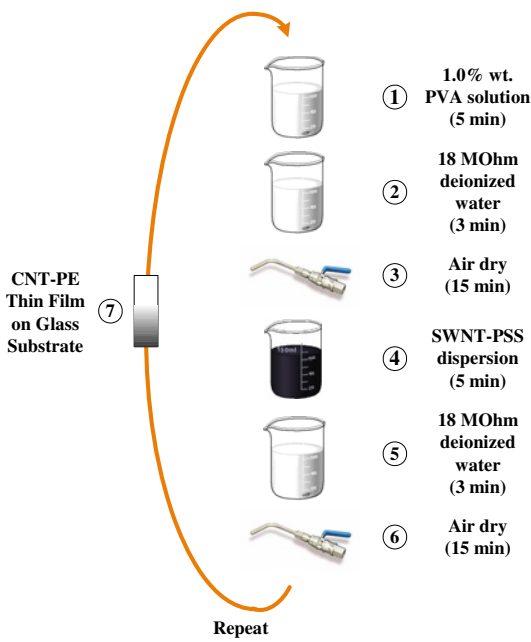

Figure 1. Illustration of the layer-by-layer deposition of one bilayer of a CNT-PE thin film using a self-assembly process.

entails the sequential dipping of a charged substrate (i.e. glass, silicon, among others) in oppositely charged polyanion and polycation solutions to deposit a variety of species one monolayer at a time (as depicted in figure 1). Selective deposition of each additional monolayer is based on opposite charge electrostatic and van der Waals force interactions. By controlling fabrication parameters, such as PE, concentration of dipping solutions, and dipping time, thin films of variable compositions can be achieved.

The first monolayer in the LbL assembly process is deposited by dipping a clean, charged glass microscope slide in a positively charged polycation solution (in this case, a PE solution such as poly(vinyl alcohol) (PVA, Sigma) or polyaniline (PANI, Aldrich)) for $5 \mathrm{~min}$. Excessively large particulates and loosely adsorbed PE species are rinsed off in $18 \mathrm{M} \Omega$ deionized water for $3 \mathrm{~min}$, followed by a drying step for $15 \mathrm{~min}$ to prevent cross-contamination between the oppositely charged solutions. Using very fast magic-angle spinning nuclear magnetic resonance (MAS NMR), Rodriguez et al [25] has verified that the adsorbed polyelectrolytes remain deposited even after rinsing. The CNT monolayer deposition is achieved by dipping the PE-coated substrate in a stable, negatively charged polyanionic CNT dispersion (CNTs from Carbon Nanotechnologies, Inc.) for $5 \mathrm{~min}$, followed by the rinsing/drying steps described above. Here, a $1.0 \mathrm{wt} \%$ aqueous solution of poly(sodium 4-styrene-sulfonate) (PSS, $1000000 M_{w}$, Aldrich) is employed to achieve a stable, negatively charged suspension of CNTs. This process completes one full cycle of the LbL assembly to form one bilayer of the CNT-PE thin film. Multilayer thin-film assembly is realized by repeating the aforementioned procedure to fabricate free-standing films of 50 and 100 bilayers of different compositions (table 1). In this study, an $n$-bilayer composite thin film fabricated with oppositely charged species $X$ and $Y$ will be denoted as $(X / Y)_{n}$. Specifically, three different CNTs are used (unpurified SWNTs (u-SWNTs), purified SWNTs (p-SWNTs), and purified DWNTs (p-DWNTs)). Also, two different PE species, PVA and PANI, are employed in the thinfilm composites for strain and corrosion sensing, respectively.

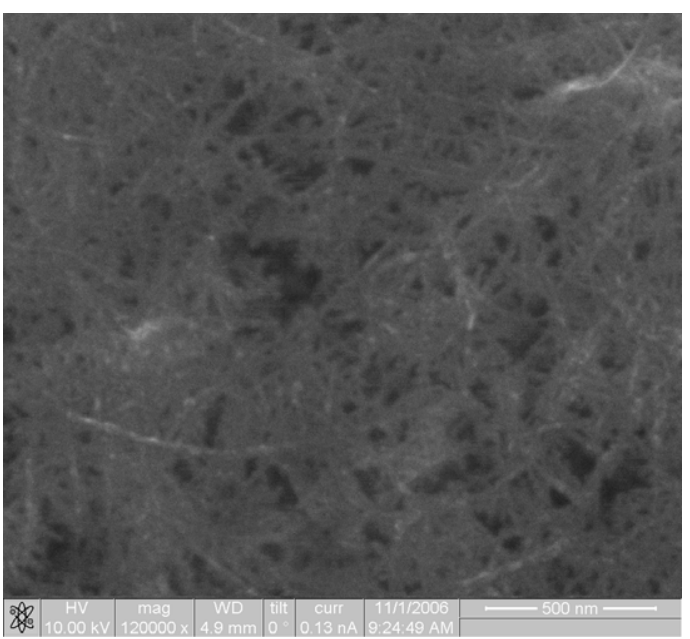

Figure 2. SEM image of (SWNT-PSS/PVA $)_{50}$ thin film, indicating adequately dispersed SWNTs deposited on the LbL thin film.

\subsection{Suspension and dispersion of carbon nanotubes}

In order to fully harness the impressive electrical properties of CNTs and to transfer these properties to tangible length scales (i.e. LbL thin films), a stable suspension and dispersion of CNTs in an aqueous solution is necessary. Currently, many researchers have undertaken covalent stabilization techniques to molecularly bind specific species to CNT surfaces for enhanced dispersion and functionality [26]; however, a noncovalent approach via steric stabilization of CNTs in polyelectrolyte solutions is selected for this study as this method preserves the mechanical and electrical properties of individual nanotubes [27]. A high-molecular-weight poly(sodium 4-styrene-sulfonate) $\left(M_{w} \approx 1000000\right)$ polyanion solution is employed to facilitate dispersion of single- and double-walled carbon nanotubes. Dispersion is achieved through $180 \mathrm{~min}$ in an ultrasonication bath $(135 \mathrm{~W}, 42 \mathrm{kHz})$ followed by $90 \mathrm{~min}$ of high-powered probe sonication $(3.178 \mathrm{~mm}$ tip, $500 \mathrm{~W}, 22.0 \mathrm{kHz})$. Adequate dispersion of CNTs is verified with scanning electron microscopy (SEM), where figure 2 shows deposition of only individual and small nanotube bundles with the percolation threshold exceeded. While it has been found in preliminary studies that PSS facilitates dispersion of SWNTs [28], Moore et al [29] validate a wide variety of polymer dispersive agents for CNTs. A key finding they report is that higher-molecular-weight polymers (e.g. PSS) tend to suspend more SWNTs due to their longer polymeric chains and the size of their hydrophilic groups for enhanced steric stabilization [29]. Nevertheless, a wide variety of polymers and surfactants has been shown to provide adequate dispersion of nanotubes in solution [30]. Preliminary UV-vis studies of CNT-PE thin films fabricated with PSS and PVA indicate greater absorbance than films fabricated with other polyelectrolytes (namely PDMA, PAH, among others). Measured UV-vis absorbance correlates to the amount of CNT deposition [31], thereby suggesting efficient nanotube deposition per LbL fabrication cycle when using PVA as the LbL electrolyte counterpart to PSS. 


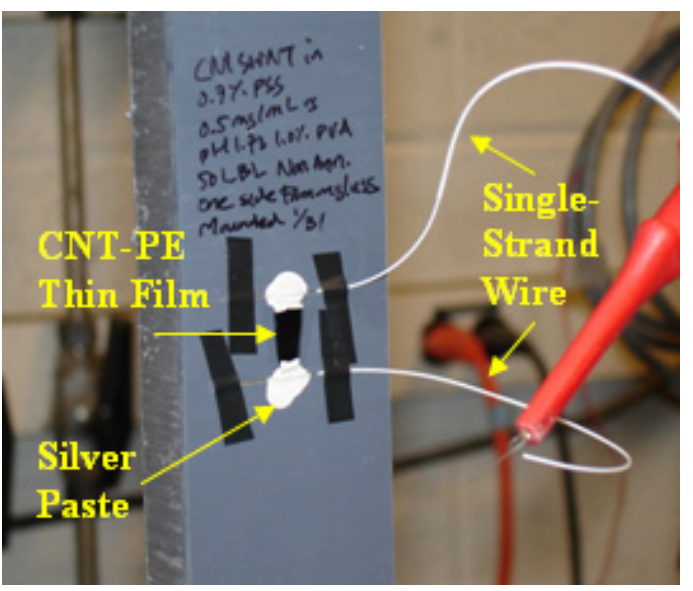

Figure 3. SWNT-PSS/PVA strain sensor on glass epoxy-bonded to a PVC specimen fixed within an MTS-810 load frame.

\subsection{CNT-PSS/PVA piezoresistive response under tensile-compressive cyclic loading}

Investigation of CNT-PE thin film piezoresistivity is conducted by applying a three-cycle tensile-compressive cyclic load pattern. First, all thin films fabricated are cut from the glass substrate into small rectangles $\left(2 \times 1 \mathrm{~cm}^{2}\right)$ and affixed to rectangular PVC coupon samples $(31 \mathrm{~cm}$ long, $4 \mathrm{~cm}$ wide, $2 \mathrm{~cm}$ thick) using CN-Y post-yield epoxy (Tokyo Sokki Kenkyujo). Electrical contacts are established by drying colloidal silver paste (Ted Pella) over single-strand wire to form a two-probe measurement setup as shown in figure 3. After a $6 \mathrm{~h}$ drying period (of the epoxy and silver paste), the coupon is loaded in an MTS-810 load frame to apply slow $\left(0.05 \mathrm{~mm} \mathrm{~s}^{-1}\right)$ and fast $\left(0.10 \mathrm{~mm} \mathrm{~s}^{-1}\right)$ saw-tooth tensile-compressive cyclic load patterns with $5000 \mu \mathrm{m} \mathrm{m}^{-1}$ strain amplitudes. Real-time resistance changes under applied loading are sampled at $1 \mathrm{~Hz}$ using an Agilent 34401A digital multimeter supplying $10 \mu \mathrm{A}$ of direct current (DC). Stroke displacement of the MTS-810 load frame is also sampled to provide a baseline comparison of bar strain with experimental CNT-PE resistivity data measured.

Earlier studies have demonstrated that SWNT-PE multilayer thin films can be tailored to a wide variety of macro-scale strain sensing properties [28]. Changes in sensitivity, hysteretic behavior, and linearity depend on various layer-by-layer fabrication parameters. First, by varying the type of CNTs used, the overall electrical characteristic of the thin film can change dramatically. It is known that SWNTs can be metallic or semiconducting; however, multi-walled carbon nanotubes are typically metallic or semi-metallic [6]. The bulk thin film can exhibit capacitive behavior with the inclusion of semiconducting SWNTs. On the other hand, semi-metallic or metallic double-walled carbon nanotubes (DWNTs) should show little or no capacitive behavior respectively. In order to experimentally investigate how changes in the type of nanotubes affect bulk strain sensitivity, three types of CNTs (i.e. unpurified SWNTs (u-SWNTs), purified SWNTs (p-SWNTs), and purified DWNTs (p-DWNTs)) are employed to fabricate multilayer CNT-PSS/PVA thick films for strain sensing.

Furthermore, since CNT-PSS/PVA thin films derive their piezoresistivity from the embedded nanotubes in the polymer matrix, the average number of CNTs deposited should directly alter strain sensing performance. By varying the concentration of CNTs in PSS solution, the average number of CNTs deposited should increase in tandem with increasing CNT concentration. Specifically, three different concentrations will be investigated: $0.25,0.50$, and $0.80 \mathrm{mg} \mathrm{ml}^{-1}$ (weight per PSS solution volume). The increase in carbon nanotube deposition creates more nanotube-to-nanotube junctions, thus providing a greater number of paths for electrical current to flow from one electrode to the other while reducing the overall resistivity of the thin film [32]. With increasing nanotube junctions, it is hypothesized that the resistive sensitivity of the thin film to applied strain will increase in tandem as a function of thickness.

Another fabrication parameter of interest is the thickness of the CNT-PSS/PVA thin films themselves. While thicker films can provide additional mechanical strength, it is hypothesized that more layers decrease the overall sensitivity of the film; clearly, a tradeoff exists between mechanical strength and electrical sensing properties. Thus, two different thicknesses, namely 50- and 100-bilayer thin films, are fabricated to identify changes in CNT-PSS/PVA thin films' electronic properties.

\subsection{CNT-PE pH sensing}

In order to realize $\mathrm{pH}$ sensing on LbL SWNT-PE thin films, a $\mathrm{pH}$-sensitive polyelectrolyte is selected as the primary matrix for purified SWNTs [19]. Polyaniline is selected to fabricate SWNT-PSS/PANI LbL thin films of 50 and 100 bilayers. Similar to section 2.3 , electrical contacts are established by drying silver paste (Ted Pella) over single-strand wire at two ends of the film. An Agilent 34401 A multimeter is employed to sample resistance change at $1 \mathrm{~Hz}$ via a two-point probe method. The $\mathrm{pH}$ sensing is detected using the thin film fitted with a small plastic well with high-vacuum grease (Dow Corning) to serve as the solution chamber. Solutions of $\mathrm{pH}$ ranging from 1 to 12 are pipetted into the chamber, while the multimeter simultaneously measures resistance changes.

\subsection{Thermal-chemical resistivity behavior}

While the layer-by-layer method is a versatile fabrication technique ideally suited for tailoring specific macro-scale sensor properties simply by adjusting initial fabrication parameters, preliminary studies have identified two effects that adversely affect sensor performance (namely an exponential decay in film resistivity and light sensitivity) [28]. The aforementioned two environmental factors are briefly discussed in the hope of identifying their origins as well as proposing a solution to mitigate their effects. First, it has been identified that CNT-PE thin films display time-variant exponential decay of bulk film resistivity [28]. While the underlying nature of this resistivity decay is unclear, CNT-PE thin films are strongly influenced by temperature, exhibiting a significant decrease in film resistance with increasing temperature $[33,34]$. When measuring thinfilm resistance under applied strain or $\mathrm{pH}$, the Agilent 34401A multimeter supplies a constant current ( $i=500 \mathrm{nA}-10 \mathrm{~mA}$ ), which may cause resistive heating in the CNT-PE sample. The direct current (DC) supply can increase the temperature of the bulk film while activating electrons at nanotube-to-nanotube 
Multifunctional layer-by-layer carbon nanotube-polyelectrolyte thin films for strain and corrosion sensing

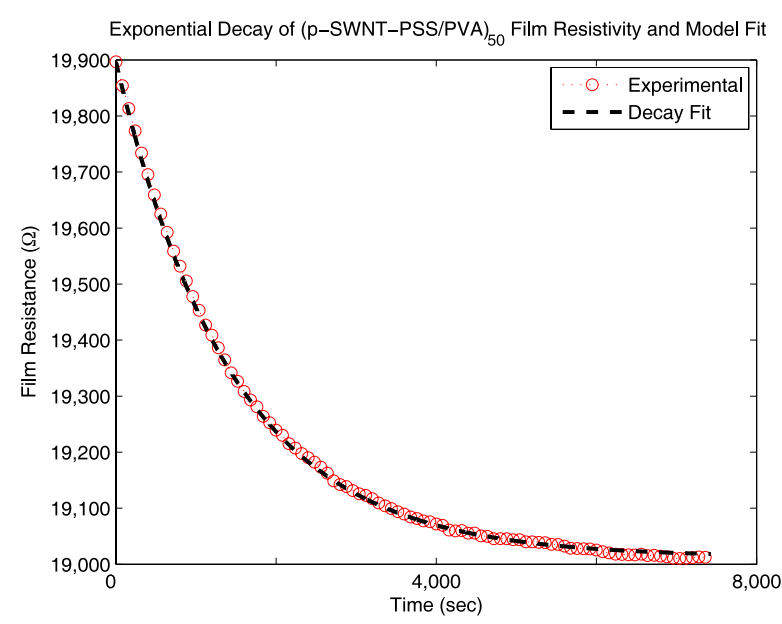

(a)

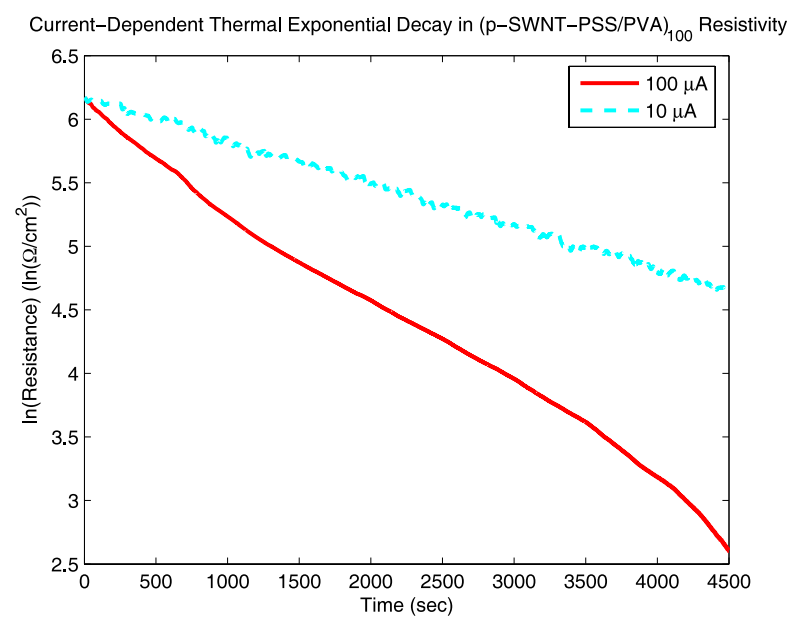

(b)

Figure 4. (a) (p-SWNT-PSS/PVA) 50 thin film exponential decay in resistance. (b) Current (thermal)-dependent bulk film resistivity decay for a (p-SWNT-PSS/PVA) $)_{100}$ thin film.

junctions which correspond to an increase in bulk film conductance. To illustrate this behavior and to quantify the evolution of film resistance as a function of time, an experiment is conducted to measure the resistivity of the thin film over a period of five hours. Valentini et al [33] have found that asgrown RF-PECVD (radio-frequency pulsed plasma enhanced chemical vapor growth) CNT thin films exhibit a negative relationship between bulk resistivity and temperature. A similar temperature-resistance relationship has been identified by Hone et al [34] using aligned and non-aligned single-walled carbon nanotube films.

\subsection{Light sensitivity}

It has been demonstrated by Zhang and Iijima [35] that bundles of as-produced carbon nanotubes display distinct deformations, including stretching, bending, and repulsion, under the illumination of visible light. The measured light-induced current shows complete elastic and recoverable behavior, as is also observed by $\mathrm{Lu}$ and Panchapakesan [36]. Furthermore, while light-induced current is insensitive to the wavelength and polarity of visible light [35], the induced photocurrent is linearly related to light intensity [36]. On the other hand, when illuminated under UV light, Chen et al [37] observe that individual carbon nanotubes exhibit orders of magnitude decrease in conductance due to intrinsic photoactivity, particularly from semiconducting SWNTs. In this study, to quantify the changes in bulk resistivity of LbL CNT-PE thin films under the illumination of light, broadspectrum visible light is shone at increasing time intervals (namely 10, 20, 40, and $60 \mathrm{~s}$ ) upon samples enclosed in a dark experimental chamber. An Agilent 34401A multimeter is again employed to sample resistance at $1 \mathrm{~Hz}$ throughout the duration of testing.

\section{Results and discussion}

\subsection{CNT-PSS/PVA thermal response}

In order to quantify the exponentially decaying bulk film resistivity due to current (from the Agilent 34401A multimeter) during sampling of resistance, the experiment described in section 2.5 is carried out for multiple film specimens. As seen from figure 4(a), CNT-PE thin films exhibit a well defined exponential decay in film resistance, $R$. Even at small input DC current sources (i.e. $5 \mu \mathrm{A}$ ), a similar effect can be observed. Nevertheless, the exponential decay in film resistance can be modeled accurately as shown in figure 4(a). By fitting the exponential decay with $R=A \mathrm{e}^{B t}+C$ (where $A, B$, and $C$ are model constants) followed by subtraction of the decay from experimental resistivity data, CNT-PE strain and corrosion sensing response can be extracted.

As mentioned in section 2.5 , it is hypothesized that one environmental factor contributing to the exponential decay of bulk film resistivity is a result of resistive heating supplied by the multimeter's DC current. Naturally, with larger applied currents, heating should be more pronounced and film resistivity should decay faster as compared to instances using smaller applied currents. Figure 4(b) plots the natural logarithm of measured film resistance (normalized to film area), as a function of time, so that film resistivity decay rates can be compared for two different DC currents (10 and $100 \mu \mathrm{A})$. As expected, a higher current $(100 \mu \mathrm{A})$ causes the temperature of the thin film to increase while causing film resistivity to decrease at a faster rate. Results from figure 4(b) confirm CNT-PSS/PVA thin film's sensitivity to temperature due to an applied current source.

\subsection{CNT-PSS/PVA thin-film light sensitivity}

Consistent with findings from Zhang and Iijima [35] and Lu and Panchapakesan [36], LbL CNT-PE thin films display decreasing resistivity upon the illumination of broad-spectrum visible light as seen from figure 5(a). Although CNT-PE thin films show their characteristic exponential decay in bulk resistance in dark settings, the incidence of light results in an immediate decrease in film resistance (figure 5(a)). As soon as the light is turned off, film resistance gradually increases to return to its initial exponentially decreasing resistivity trend. By fitting the exponential decay with $R=$ $A \mathrm{e}^{B t}+C$ and then subtracting it from the experimental data, 
(DWNT-PSS/PVA) ${ }_{50}$ Resistance vs. Time Under Intervals of llluminated Light

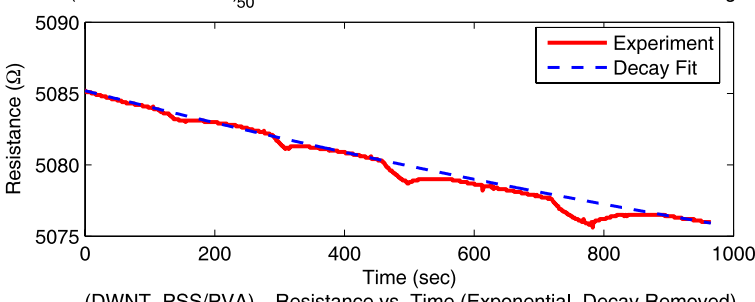

(DWNT-PSS/PVA) ${ }_{50}$ Resistance vs. Time (Exponential-Decay Removed)

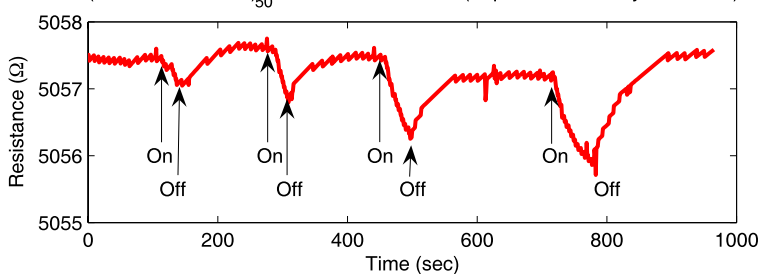

Figure 5. (a) Top, (p-DWNT-PSS/PVA) $)_{50}$ thin film response to broad-spectrum visible light at 10,20,40, and $60 \mathrm{~s}$ intervals along with the fitted exponential decay of film resistivity; (b) bottom, exponential decay removed thin-film light response.
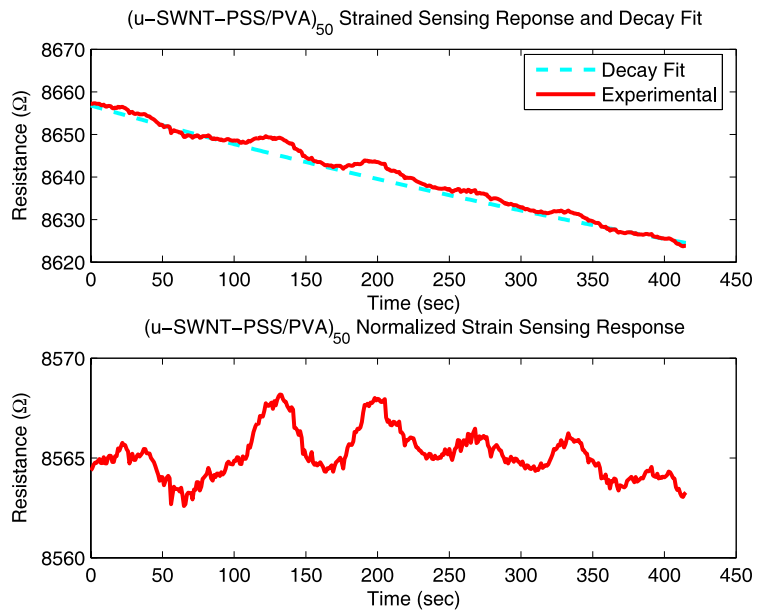

Figure 6. (a) Top, (u-SWNT-PSS/PVA) $)_{50}$ thin-film response to a three-cycle tensile-compressive load pattern to $\varepsilon=5000 \mu \mathrm{m} \mathrm{m}^{-1}$; (b) bottom, exponential decay removed strain sensing response suggests thin-film piezoresistivity cannot be accurately extracted.

the equivalent CNT-PE light-sensitive response is extracted (figure 5(b)). From figure 5(b), it is clear that as the 'light on' interval is increased the associated drop in resistance increases accordingly. Given sufficient time after light is removed from the sample, resistivity recovery is fully elastic and complete. Although figure 5 is of a (DWNT-PSS/PVA $)_{50}$ sample, similar results have been observed for SWNT-PSS/PVA LbL thin films. In order to prevent light from interfering with measured data, strain and corrosion sensing experiments are conducted by enclosing samples in a dark environment.

\subsection{CNT-PSS/PVA strain sensing response}

Previous studies conducted with SWNT-PE films fabricated with PSS and PVA polyelectrolyte conjugates have verified their piezoresistive response [28]. Here, the experimental CNT-PSS/PVA (CNTs considered include u-SWNTs, pSWNTs, and p-DWNTs) thin-film response under applied
$(\mathrm{p}-\mathrm{DWNT}-\mathrm{PSS} / \mathrm{PVA})_{50}$ Strain Sensing Response and Decay Fit

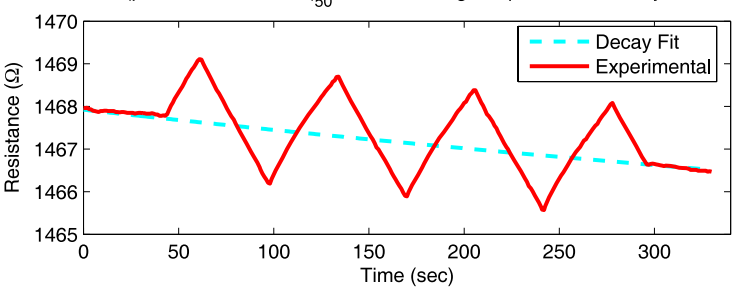

$(\mathrm{p}-\mathrm{DWNT}-\mathrm{PSS} / \mathrm{PVA})_{50}$ Normalized Strain Sensing Response

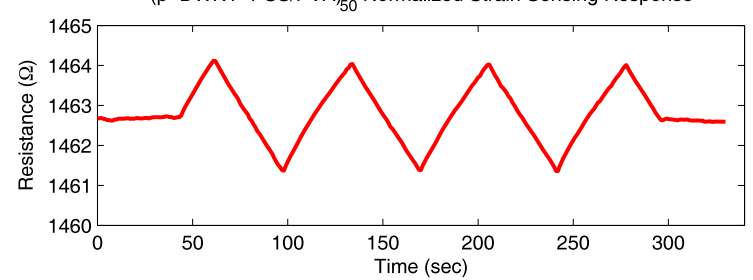

Figure 7. (a) Top, (DWNT-PSS/PVA) $)_{50}$ thin-film response to a three-cycle tensile-compressive load pattern to $\varepsilon=5000 \mu \mathrm{m} \mathrm{m}^{-1}$; (b) bottom, exponential decay removed strain sensing response.

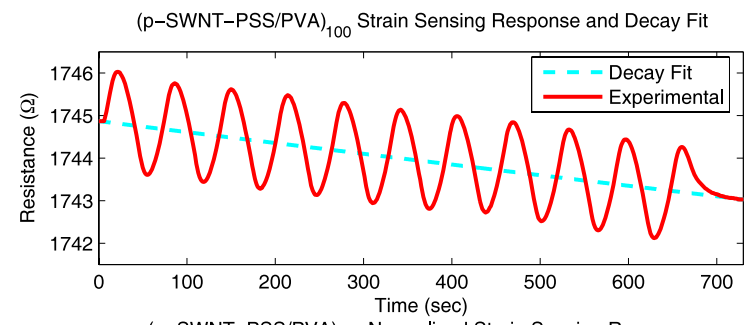

$(p-S W N T-P S S / P V A){ }_{100}$ Normalized Strain Sensing Response

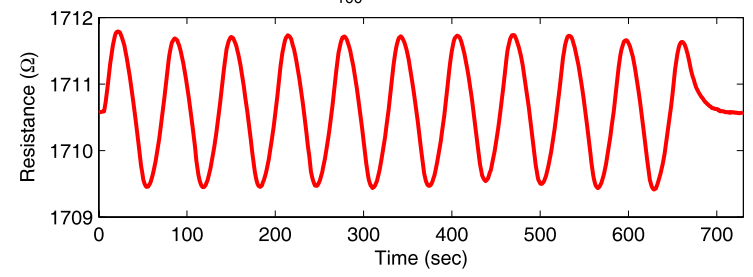

Figure 8. (a) Top, (SWNT-PSS/PVA) $)_{100}$ thin-film response to a ten-cycle tensile-compressive load pattern to $\varepsilon=5000 \mu \mathrm{m} \mathrm{m}^{-1}$; (b) bottom, exponential decay removed strain sensing response.

tensile-compressive strains is used to identify bulk strain sensing and inherent film properties (table 1). Most importantly, the strain sensitivity for the strain sensor (denoted as $S_{R}$ ) is expressed as

$$
S_{R}=\frac{\Delta R_{\text {peak-peak }} / R_{0}}{\varepsilon_{\text {peak }- \text { peak }}}
$$

where $\varepsilon=\Delta L / L_{0}, \Delta L$ is the change in length of the PVC element, and $L_{0}$ is the unsupported length of the PVC bar; $\Delta R_{\text {peak-peak }}$ is defined as the change in film resistance between maximum and minimum applied strains; and $R_{0}$ is the nominal resistance of the thin film. Representative u-SWNT-, pDWNT- and p-SWNT-PSS/PVA multilayer film piezoresistive responses are shown in figures 6(a), 7(a), and 8(a). Upon identifying the resistivity decay model for each of the 18 different films (first three rows of table 1), the exponential fit is numerically removed to determine film strain sensitivity, $S_{R}$ (figures 6(b), 7(b), and 8(b)). Excellent model fitting can 


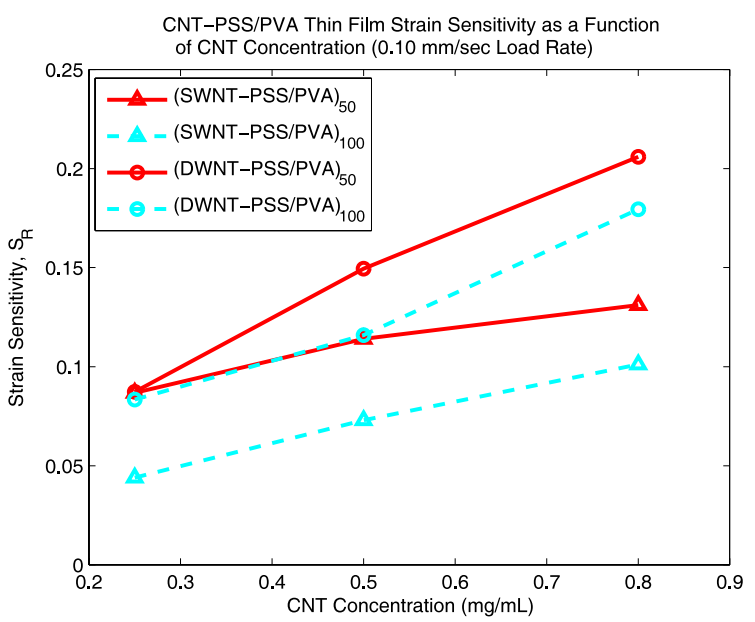

(a)

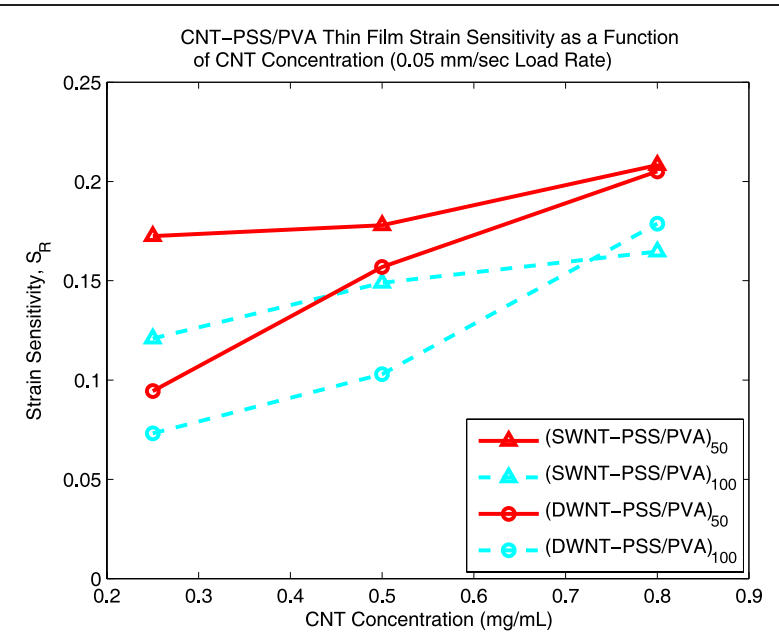

(b)

Figure 9. Strain sensitivity for different CNT-PSS/PVA thin films as a function of SWNT concentration $\left(0.10 \mathrm{~mm} \mathrm{~s}^{-1}\right.$ load rate); (b) strain sensitivity for different CNT-PE thin films as a function of SWNT concentration $\left(0.05 \mathrm{~mm} \mathrm{~s}^{-1}\right.$ load rate).

Table 2. Calculated p-SWNT- and p-DWNT-PSS/PVA thin-film strain sensitivities, $S_{R}$ (the first number denotes strain sensitivity for a testing load rate of $0.10 \mathrm{~mm} \mathrm{~s}^{-1}$ and the second for a load rate of $0.05 \mathrm{~mm} \mathrm{~s}^{-1}$ ).

\begin{tabular}{llll}
\hline & $0.25 \mathrm{mg} \mathrm{ml}^{-1} \mathrm{CNT}$ & $0.50 \mathrm{mg} \mathrm{ml}^{-1} \mathrm{CNT}$ & $0.80 \mathrm{mg} \mathrm{ml}^{-1} \mathrm{CNT}$ \\
\hline (p-SWNT-PSS/PVA $_{50}$ & $0.087 / 0.173$ & $0.114 / 0.178$ & $0.131 / 0.208$ \\
(p-SWNT-PSS/PVA) $_{100}$ & $0.044 / 0.121$ & $0.073 / 0.149$ & $0.101 / 0.165$ \\
(p-DWNT-PSS/PVA) & $0.087 / 0.094$ & $0.150 / 0.157$ & $0.206 / 0.205$ \\
(p-DWNT-PSS/PVA) $_{100}$ & $0.083 / 0.073$ & $0.116 / 0.103$ & $0.179 / 0.179$ \\
\hline
\end{tabular}

be observed, as the fit removed results show repeatable strain sensing response with no resistivity drifts.

Although all three types of CNT-PSS/PVA thin films exhibit piezoresistive response, u-SWNT-PSS/PVA thin films possess a high noise floor, making them unsuitable for strain sensing (figure 6). Equivalent strain sensitivities could not be extracted accurately due to excessive noise masking the response to applied tensile-compressive strains. Despite the fact that u-SWNTs have a large quantity of impurities (e.g. amorphous carbon, iron, nickel, among others), their high noise floor may be due to premature clumping during LbL fabrication as opposed to the impurities on individual nanotubes. Efforts to disperse u-SWNTs in $1.0 \%$ PSS solutions have been successful; however, precipitation occurs significantly faster than solutions of p-SWNTs and p-DWNTs. As a result, increasingly large bundles of u-SWNTs are deposited during each additional LbL bilayer fabrication cycle. Thus, for the remainder of this study, performance characterization of LbL CNT-PSS/PVA thin films will only be focused on those fabricated with purified nanotubes.

Between p-DWNT- (figure 7) and p-SWNT-PSS/PVA (figure 8) thin films, an obvious difference is observed. Here, p-DWNT-PSS/PVA thin films exhibit sharp peaks at points of maximum applied tensile-compressive strains, whereas rounded peaks are identified for p-SWNT-PSS/PVA samples. Although the origins of these two different types of responses are unknown, it is hypothesized that the existence of significantly more semiconducting nanotubes in SWNTs contributes to such behavior. Ongoing studies in our laboratory are focusing on studying the potential capacitive behavior of the CNT-PE thin films by using frequency-domain characterization techniques such as electrical impedance spectroscopy.

Among the various films tested, strong trends can be identified between strain sensor sensitivity and CNT concentration (table 2). First, as CNT concentration is increased from 0.25 to $0.80 \mathrm{mg} \mathrm{ml}^{-1}$, the strain sensitivity of the thin film increases, irrespective of the number of layers; this can be observed by considering each row of table 2 and figure 9. Stadermann et al [32] shows that as CNT content is increased the local conductance of the thin film relies heavily on the number of nanotube-to-nanotube junctions. The increased nanotube junctions present in higher-CNTconcentration thin films allow for higher sensitivity to changes in applied strain as the films are stretched or compressed. In addition, this trend applies to films fabricated with different thicknesses and types of carbon nanotubes (i.e. p-SWNTs and p-DWNTs) and is consistent between the two different load rates of 0.10 and $0.05 \mathrm{~mm} \mathrm{~s}^{-1}$.

Second, as film thickness is increased from 50 to 100 bilayers, thin-film strain sensitivity of the same type of CNT progressively decreases. Yet, experiments conducted with free-standing LbL films suggest an increase in mechanical strength and ultimate strain capacity with increasing film thickness. Here, a tradeoff exists between optimizing CNTPSS/PVA thin films for higher sensitivity at the cost of lower ultimate strain capacity or vice versa. Although the highest calculated strain sensitivity obtained in this set of experiments is $S_{R}=0.208$, an order of magnitude higher strain sensitivity has been achieved in other related studies. Enhancement 

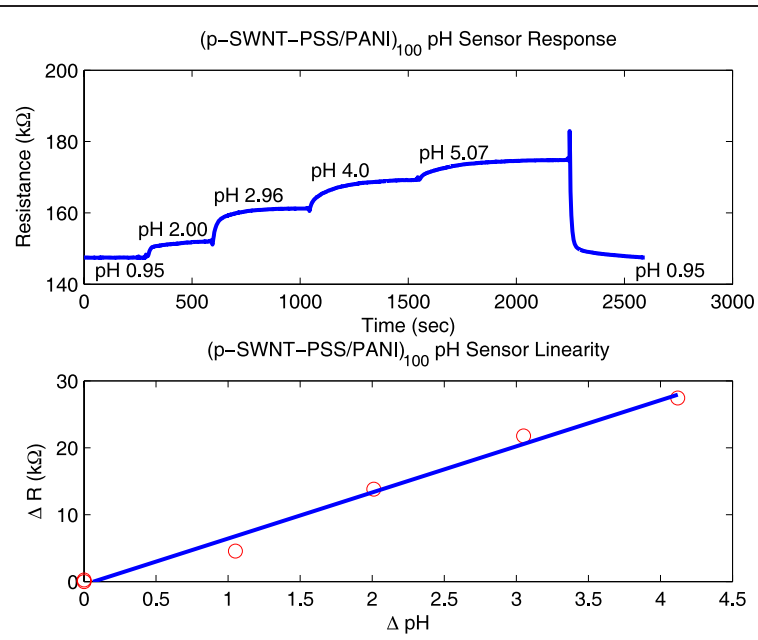

Figure 10. (a) Top, (p-SWNT-PSS/PANI) 100 acidic solution (pH 0.95-5.07) $\mathrm{pH}$ sensing response; (b) bottom, normalized change of resistance as a function of $\mathrm{pH}$, indicating linearity of $\mathrm{pH}$ sensor (sensitivity, $S_{P}=4.56 \mathrm{k} \Omega \mathrm{cm}^{-2} / \mathrm{pH}$ ).

of thin-film strain sensitivity while preserving mechanical strength (thicker LbL films) may be achieved by increasing LbL dipping time as had been done in previous studies where higher strain sensitivity has been attained. Comparing with recently proposed CNT-based strain sensors, LbL CNT-PE thin film strain sensitivity appears to be an order of magnitude lower than those commonly reported [13]. A strain sensor sensitivity of up to 7 for a buckypaper specimen formed with $100 \%$ SWNTs has been reported by Kang et al [13]; however, buckypaper specimens show nonlinear behavior above $\varepsilon=$ $500 \mu \mathrm{m} \mathrm{m}^{-1}$. Furthermore, the mechanically strong LbL thin films can be tailored to different strain sensitivities while exhibiting linear performance up to $\varepsilon=10000 \mu \mathrm{m} \mathrm{m}^{-1}$.

Finally, between p-SWNT- and p-DWNT-based LbL strain sensors fabricated with the same CNT concentration, no obvious trend can be identified. However, a comparison between figures 9(a) and (b) suggests p-SWNT-PSS/PVA thin films are more susceptible to load-rate-dependent effects, whereas p-DWNT-PSS/PVA thin films exhibit similar strain sensitivities for both the fast and slow load rates $(0.10$ and $0.05 \mathrm{~mm} \mathrm{~s}^{-1}$, respectively). This load-rate-dependent effect may be due to capacitive behavior observed in the time-domain resistivity plots (figure 8 ).

In general, under applied strains, CNTs displace in the bulk polymer matrix or deform by stretching, shrinking, or bending. Assuming an applied tensile strain, CNTs can undergo rigid body motion due to stretching of the bulk film while decreasing the number of nanotube-to-nanotube junctions, thereby increasing bulk film resistivity (the opposite applies for compression). For the latter case (decreased number of junctions), deformations due to applied strain alter the inherent conductivity of individual nanotubes [11-13]. However, in the case of DWNT thin films (or MWNT), Qian and Dickey [38] report that when MWNTs are nearly parallel to the direction of applied tensile strain, a "swordin-sheath' atomic fracture failure is preferred with an inner tube individually translating relative to the outer tube. Due to the weak interlayer bonding of MWNTs, applied strain can induce some concentric rings of individual MWNTs to rupture
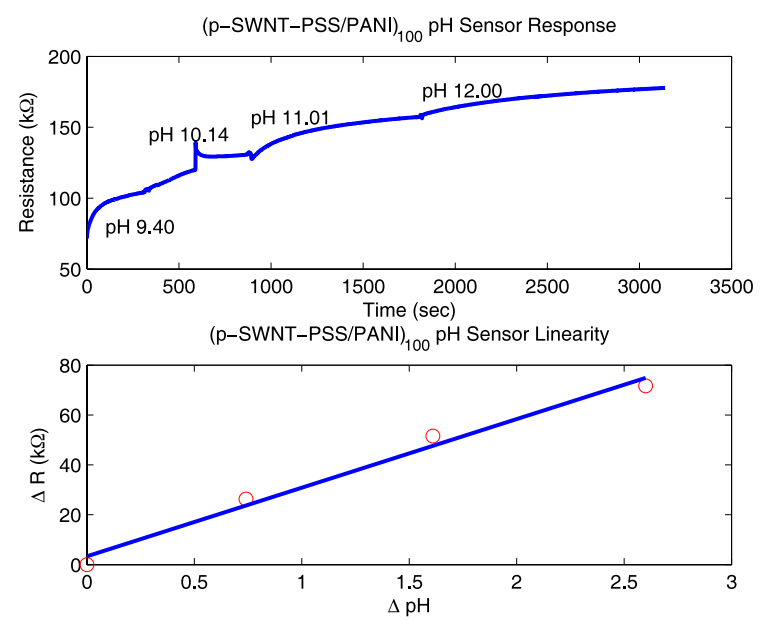

Figure 11. (a) Top, (p-SWNT-PSS/PANI) 100 alkaline solution $(\mathrm{pH}$ 9.40-12.00) $\mathrm{pH}$ sensing response; (b) bottom, normalized change of resistance as a function of $\mathrm{pH}$, indicating linearity of $\mathrm{pH}$ sensor (sensitivity, $S_{P}=20.66 \mathrm{k} \Omega \mathrm{cm}^{-2} / \mathrm{pH}$ ).

first [38]. This premature failure mechanism at lower strains can reduce the overall bulk strain sensitivity of p-DWNTPSS/PVA thin films as observed in figure 9. Upon a 'sword-insheath' fracture failure, inner and outer tubes can lose electrical connectivity as the inner and outer tubes separate from one another due to the applied strain.

\section{4. $p$-SWNT-PSS/PANI $p H$ sensing response}

As mentioned in section 2.4, p-SWNT-PSS/PANI thin films are employed as proof of concept in this $\mathrm{pH}$ sensing validation study. While this study may appear to coincide with work conducted by Kaempgen and Roth [19], where they have presented an excellent CNT/PANI pH sensor of high sensitivity, p-SWNT-PSS/PANI thin-film $\mathrm{pH}$ sensors show potential for tailoring them to specific $\mathrm{pH}$ sensitivities and range. By employing the versatile $\mathrm{LbL}$ fabrication technique, it is anticipated that initial fabrication parameters can significantly alter the performance characteristics of $p$ SWNT-PSS/PANI pH sensors.

Nevertheless, from figures 10(a) and 11(a), p-SWNTPSS/PANI exhibits high sensitivity to changing $\mathrm{pH}$ such that bulk film resistance increases progressively as $\mathrm{pH}$ is increased in tandem. By exposing p-SWNT-PSS/PVA thin films to increasing-pH solutions, one can observe drastic linear changes in film resistivity. Furthermore, $\mathrm{pH}$ sensing is viable for both acidic and alkaline solutions ranging from $\mathrm{pH}$ values of 0.95 to 12.00. Similar to strain sensitivity, $\mathrm{pH}$ sensitivity $\left(S_{P}\right)$ can be calculated via equation (2).

$$
S_{P}=\frac{\Delta R / A}{\Delta \mathrm{pH}}
$$

where $\Delta R$ is the change in resistance when the film is in contact with a solution and $A$ is the surface area of the thin film. By plotting the change in resistance $(\Delta R)$ versus changes in $\mathrm{pH}$ and then normalizing by film surface area, the degree of linearity is determined. With regards to acidic $\mathrm{pH}$ sensing, figure 10(b) shows a desirable strong linearity 
between normalized changes in film resistance and changes in $\mathrm{pH}$, while its corresponding $\mathrm{pH}$ sensitivity is calculated to be $S_{P \text { acidic }}=4.56 \mathrm{k} \Omega \mathrm{cm}^{-2} / \mathrm{pH}$. However, when the $\mathrm{p}$ SWNT-PSS/PANI thin film is exposed to alkaline solutions, its response is slightly more nonlinear (figure 11(a)) but with an estimated $\mathrm{pH}$ sensitivity of $S_{P \text { alkaline }}=20.66 \mathrm{k} \Omega \mathrm{cm}^{-2} / \mathrm{pH}$ (figure 11(b)). In all cases, the proposed $\mathrm{pH}$ sensor can be 'reset' back to its nominal resistance $\left(R_{0}\right)$ simply by exposing the sensor to an acidic solution $(\mathrm{pH}=0.95)$ as shown in figure 10 (a).

Although the response of the aforementioned p-SWNTPSS/PANI pH sensor does not possess fast response time and linearity as reported by Kaempgen and Roth [19], the LbL fabrication technique allows one to tailor the sensing response simply by altering initial fabrication parameters. These preliminary results suggest great potential for utilizing LbL to fabricate $\mathrm{pH}$ sensors of specific properties. In the near future, optimization of $\mathrm{pH}$ sensing using $\mathrm{p}$-SWNT-PSS/PANI will be investigated with rate of response and linearity of the p-SWNT-PSS/PANI film considered.

\section{Conclusions}

In summary, this paper demonstrates the viability to tailor and fabricate multifunctional carbon nanotube-polyelectrolyte composite thin-film sensors for strain and corrosion monitoring. A layer-by-layer assembly method is employed to fabricate a diverse suite of CNT-PE thin films composed of varying combinations of types of CNT, types of PE, and CNT concentration. The purpose of this study is to demonstrate the versatility of the LbL method to tailor different sensing transducers (namely for strain and corrosion) simply by varying initial LbL parameters (namely the type of PE used). First, by using CNTs dispersed in PSS solution and PVA as the LbL counterpart, piezoresistive thin films can be realized. With increasing CNT concentration (an LbL fabrication parameter), one can progressively increase the strain sensitivity of the macro-scale multilayer film. Yet, a tradeoff exists between higher mechanical strength (thicker films) and lower strain sensitivity.

To produce a sensor for monitoring corrosion environments, namely $\mathrm{pH}, \mathrm{p}$-SWNT-PSS solutions coupled with PANI show excellent response to solutions of varying $\mathrm{pH}$ values (ranging from 1 to 12), where $\mathrm{pH}$ sensitivities up to $S_{P}=$ $20.66 \mathrm{k} \Omega \mathrm{cm}^{-2} / \mathrm{pH}$ have been achieved. Although CNT-PE thin films suffer from light sensitivity and a time-variant thermal exponential decay in bulk resistivity, methods have been proposed to mitigate these unwanted effects. In addition, CNT$\mathrm{PE}$ thin films are extremely sensitive to the illumination of broad-spectrum visible light, but changes in film resistivity are fully recoverable upon removing the light source. On the other hand, the exponential decay in film resistivity is found to be inherent to all CNT-PE thin films. This effect can be modeled accurately by fitting an exponential curve of the form $R=A \mathrm{e}^{B t}+C$ to numerically remove the exponential decay.

In the near future, electrical impedance spectroscopy (EIS) will be employed as a robust technique to characterize CNTPE thin films fabricated with different LbL parameters. It is hypothesized that EIS can accurately identify an equivalent circuit model from a frequency domain perspective. Upon identification of film capacitance, CNT-PE thin films can be patterned with focused ion beam (FIB) lithography into inductive coil antennas for wireless strain and corrosion sensing. Any inherent changes in resistance and/or capacitance due to environmental changes will yield characteristic frequency shifts and bandwidth changes that can be correlated to applied strain or change in $\mathrm{pH}$.

\section{Acknowledgments}

This research is funded by the National Science Foundation (grant number CMS-0528867). The authors would like to express their gratitude to Professor Victor Li and the ACEMRL group for allowing us to use their MTS-810 load frame during the experimental phase of this study.

\section{References}

[1] Iijima S 1991 Helical microtubules of graphitic carbon Nature $35456-8$

[2] Thostenson E T and Chou T-W 2002 Aligned multi-walled carbon nanotube-reinforced composites: processing and mechanical characterization J. Phys. D: Appl. Phys. 35 L 77-80

[3] Tsukagoshi K, Yoneya N, Uryu S, Aoyagi Y, Kanda A, Ootuka Y and Alphenaar B W 2002 Carbon nanotube devices for nanoelectronics Physica B 323 107-14

[4] Kong J, Franklin N R, Zhou C, Chapline M G, Peng S, Cho K and Dai H 2000 Nanotube molecular wires as chemical sensors Science $\mathbf{2 8 7} 622-5$

[5] Wood J R, Zhao Q, Frogley M D, Meurs E R, Prins A D, Peijs T, Dunstan D J and Wagner H D 2001 Carbon nanotubes: from molecular to macroscopic sensors Phys. Rev. B $627571-5$

[6] Baughman R H, Zakhidov A A and de Heer W A 2002 Carbon nanotubes - the route towards applications Science $297787-92$

[7] Salvetat J-P, Bonard J-M, Thomson N H, Kulik A J, Forro L, Benoit W and Zuppiroli L 1999 Mechanical properties of carbon nanotubes Appl. Phys. A $69255-60$

[8] Stein P K 20061936 A banner year for strain gages and experimental stress analysis-an historical perspective Exp. Tech. 30 23-41

[9] Peng S, O'Keeffe J, Wei C, Cho K, Kong J, Chen R, Franklin N and Dai H 2001 Carbon nanotube chemical and mechanical sensors Proc. 3rd Int. Workshop on Structural Health Monitoring pp 1-8

[10] Minot E D, Yaish Y, Szaonova V, Park J-Y, Brink M and McEuen P L 2003 Tuning carbon nanotube bad gaps with strain Phys. Rev. Lett. 90156401

[11] Tombler T W, Zhou C, Alexseyev L, Kong J, Dai H, Liu L, Jayanthi C S, Tang M and Wu S-Y 2000 Reversible electromechanical characteristics of carbon nanotubes under local-probe manipulation Nature 405 769-72

[12] Dharap P, Li Z, Nagarajaiah S and Barrera E V 2004 Nanotube film based on single-wall carbon nanotubes for strain sensing Nanotechnology 15 379-82

[13] Kang I, Schulz M, Kim J H, Shanov V and Shi D 2006 A carbon nanotube strain sensor for structural health monitoring Smart Mater. Struct. 15 737-48

[14] Baldini F 1998 Critical review of $\mathrm{pH}$ sensing with optical fibres Chemical, Biochemical, and Environmental Fiber Sensors X, Proc. SPIE $35402-9$

[15] Sawada K, Mimura S, Tomita K, Nakanishi T, Tanabe H, Ishida M and Ando T 1999 Novel CCD-based pH imaging sensor IEEE Trans. Electron Devices 46 1846-9

[16] Hizawa T, Sawada K, Takao H and Ishida M 2006 Fabrication of a two-dimensional $\mathrm{pH}$ image sensor using a charge transfer technique Sensors Actuators B 117 509-15 
[17] Kwon J-H, Lee K-S, Lee Y-H and Ju B-K 2006 Single-wall carbon nanotube-based $\mathrm{pH}$ sensor fabricated by the spray method Electrochem. Solid-State Lett. 9 H85-7

[18] Carino N J 1999 Nondestructive techniques to investigate corrosion status in concrete structures J. Perform. Construct. Facilities 13 96-106

[19] Kaempgen M and Roth S 2006 Transparent and flexible carbon nanotube/polyaniline $\mathrm{pH}$ sensor J. Electroanal. Chem. $58672-6$

[20] Li Z, Dharap P, Nagarajaiah S, Barrera E V and Kim J D 2004 Carbon nanotube film sensors Adv. Mater. 16 640-3

[21] Mamedov A A, Kotov N A, Prato M, Guldi D M Wicksted J P and Hirsch A 2002 Molecular design of strong single-wall carbon nanotube/polyelectrolyte multilayer composites Nat. Mater. 1 190-4

[22] Decher G 1997 Fuzzy nanoassemblies toward layered polymeric multicomposites Science 277 1232-7

[23] Kotov N A 2001 Ordered layered assemblies of nanoparticles MRS Bull. 26 992-7

[24] Paloniemi H, Lukkarinen M, Aaritalo T, Areva S, Leiro J, Heinonen M, Haapakka K and Luddari J 2006 Layer-by-layer electrostatic self-assembly of single-wall carbon nanotube polyelectrolytes Langmuir 22 74-83

[25] Rodriguez L N J, De Paul S M, Barrett C J, Raven L and Spiess H W 2000 Fast magic-angle spinning and double-quantum $\mathrm{H}$ solid state NMR spectroscopy of polyelectrolyte multilayers Adv. Mater. 12 1934-8

[26] Xie X-L, Mai Y-W and Zhou X-P 2005 Dispersion and alignment of carbon nanotubes in polymer matrix: a review Mater. Sci. Eng. R 49 89-112

[27] Tan Y and Resasco D E 2005 Dispersion of single-walled carbon nanotubes of narrow diameter distribution J. Phys. Chem. B 109 14454-60

[28] Loh K J, Lynch J P and Kotov N A 2006 Mechanical-electrical characterization of carbon nanotube thin films for structural monitoring applications 13th Ann. Int. Symp. on Smart Structures and Materials; Proc. SPIE $617461741 Z$

[29] Moore V C, Strano M S, Haroz E H, Hauge R H and Smalley R E 2003 Individually suspended single-walled carbon nanotubes in various surfactants Nano Lett. 3 1379-82

[30] Decher G and Schlenoff J B 2003 Multilayer Thin Films: Sequential Assembly of Nanocomposite Materials (Mörlenbach: Wiley-VCH)

[31] Tan Y and Resasco D E 2005 Dispersion of single-walled carbon nanotubes of narrow diameter distribution $J$. Phys. Chem. B 109 14454-60

[32] Stadermann M et al 2004 Nanoscale study of conduction through carbon nanotube networks Phys. Rev. B 69201402

[33] Valentini L, Armentano I, Puglia D, Lozzi L, Santucci S and Kenny J M 2004 A deeper understanding of the photodesorption mechanism of aligned carbon nanotube thin films by impedance spectroscopy Thin Solid Films 449 105-12

[34] Hone J, Liaguno M C, Nemes N M, Johnson A T, Fischer J E, Walters D A, Casavant M J, Schmidt J and Smalley R E 2000 Electrical and thermal transport properties of magnetically aligned single wall carbon nanotube films Appl. Phys. Lett. 77 666-8

[35] Zhang Y and Iijima S 1999 Elastic response of carbon nanotube bundles to visible light Phys. Rev. Lett. 82 3472-5

[36] Lu S and Panchapakesan B 2006 Photoconductivity in single wall carbon nanotube sheets Nanotechnology 17 1843-50

[37] Chen R J, Franklin N R, Jing K, Jien C and Tombler T W 2001 Molecular photodesorption from single-walled carbon nanotubes Appl. Phys. Lett. 79 2258-60

[38] Qian D and Dickey E C 2001 In situ transmission electron microscopy studies of polymer-carbon nanotube composite deformation J. Microsc. 204 37-45 